\title{
CONTROLLABILITY AND OBSERVABILITY OF LINEAR DISCRETE-TIME FRACTIONAL-ORDER SYSTEMS
}

\author{
SAID GUERMAH *，SAid DJENNOUnE*，MAAmar BETTAYEB ** \\ * Laboratoire de Conception et Conduite des Systèmes de Production \\ Universitè Mouloud Mammeri de Tizi-Ouzou, BP 17 RP, Tizi-Ouzou, Algeria \\ e-mail: \{saidguermah,s_djennoune\}@yahoo.fr \\ ** Electrical \& Computer Engineering Department \\ University of Sharjah, P.O. Box 27272, Sharjah, United Arab Emirates \\ e-mail:maamar@sharjah.ac.ae
}

\begin{abstract}
In this paper we extend some basic results on the controllability and observability of linear discrete-time fractional-order systems. For both of these fundamental structural properties we establish some new concepts inherent to fractional-order systems and we develop new analytical methods for checking these properties. Numerical examples are presented to illustrate the theoretical results.
\end{abstract}

Keywords: system modeling, discrete fractional state-space systems, reachability, controllability, observability, controllability and observability Gramians.

\section{Introduction}

Nowadays the concept of non-integer derivative and integral is used increasingly to model the behavior of real systems in various fields of science and engineering. Originally, fractional calculus was the field of mathematical analysis aiming at the investigation of integrals and derivatives of arbitrary orders. This topic is somewhat ancient, since it started from some speculations of G.W. Leibnitz, L'Hopital (1695, 1697), and L. Euler (1730). In a letter to L'Hopital in 1695, Leibnitz raised the following question: Can the meaning of derivatives with integer order $\mathrm{d}^{n} y(t) / \mathrm{d} x^{n}$ be generalized to derivatives with noninteger order, so that in the general case $n \in \mathbb{C}$ ?. Later, up to the middle of the 20th century, a long list of mathematicians provided important contributions to this topic, among them Laplace, Fourier, Abel, Liouville, Riemann, Grünwald, Letnikov, Hadamard, Lévy, Marchaud, Love, and Riesz.

Further steps in the development of this discipline were the organization of specialized conferences and the publication of treatises only three decades ago. The first of such conferences-it is a milestone-was organized by B. Ross in 1974.
Recent books (Oldham and Spanier, 1974; Oustaloup, 1983; Samko et al., 1993; Miller and Ross, 1993; Oustaloup, 1995; Gorenflo and Mainardi, 1997; Podlubny, 1999; Kilbas et al., 2006) provide a rich source of references on fractional-order calculus.

In the particular domain of control theory, several authors have been interested by this aspect since the 1960s. The first contributions (Axtell and Bise, 1990; Manabe, 1960; Oustaloup, 1983) provided generalizations of classical analysis methods for fractional-order systems (transfer function, frequency response, pole and zero analysis, etc.).

Recently, this tool has come into current use for modeling physical phenomena of real systems such as electrochemistry (Ichise et al., 1971), electromagnetism and electrical machines (Lin et al., 2000), thermal systems and heat conduction (Battaglia et al., 2001; Cois et al., 2002), transmission and acoustics (Matignon, 1994; Matignon et al, 1994), viscoelastic materials (Hanyga, 2003), and robotics (Valerio and Costa, 2004). These systems exhibit hereditarily properties and long memory transients, which can be represented more accurately by fractionalorder models. 
Consequently, numerous contributions are regularly resorting to the fractional-order aspect in system modeling, namely, with state space representation, in parameter estimation, identification, and controller design. The CRONE approach (in French: Commande Robuste d'Ordre Non Entier) was elaborated to offer new solutions to control problems, such as vehicle suspension. Two noticeable conferences (the 41st IEEE CDC conference in 2002 and the IFAC FDA'04 workshop in 2004) treated the application of fractional-order calculus to automatic control theory.

The state-space representation of fractional-order systems was introduced in (Raynaud and Zergainoh, 2000; Hotzel and Fliess, 1998; Dorčák et al., 2000; Sabatier et al., 2002; Vinagre et al., 2002). It emerged that for fractional-order systems, two different interesting types can be considered: the commensurate-order and the non-commensurate-order systems. The system is of a commensurate-order if all the orders of derivatives are multiple integers of a base $\alpha$, where $\alpha$ denotes the differentiation order. The state-space representation was exploited in the analysis of system performances. In fact, the solution of the state-space equation was derived by using the Mittag-Lefller function (Mittag-Leffler, 1904). The stability of the fractional-order system was investigated (Matignon and d'Andrèa Novel, 1996). A condition based on the principle of the argument was established to guarantee the asymptotic stability of the fractional-order system. Further, controllability and observability properties were defined, and some algebraic criteria of these two properties were derived (Matignon and d'Andrèa Novel, 1996).

A contribution to the analysis of the controllability and observability of commensurate continuous-time fractional-order systems modeled by fractional state space equations was recently made in (Bettayeb and Djennoune, 2006).

Linear discrete-time fractional-order systems modeled by a state space representation were introduced in (Dzieliński and Sierociuk, 2005; Dzieliński and Sierociuk, 2006; Dzieliński and Sierociuk, 2007). These contributions are devoted to controllability and observability analysis, the design of a Kalman filter and observers, plus adaptive feedback control for discrete fractionalorder systems.

Our objective in the present paper is to contribute to the analysis of the controllability and observability of linear discrete-time fractional-order systems. To the best of our knowledge, controllability as well as some aspects of the observability of such systems have not been treated yet. Two reviewers brought to our attention the paper (Dzieliński and Sierociuk, 2007), which appeared several months after we submitted the present paper. This work is complementary to ours. We propose new concepts that are inherent to fractional-order systems, and we establish te- stable sufficient conditions for guaranteeing the existence of these structural properties.

The remainder of this paper is organized as follows: In Section 2, we recall some fundamental definitions of fractional derivatives and fractional-order systems, modeled by continuous models. Then we expose a discrete-time model, as defined in (Dzieliński and Sierociuk, 2005), and we introduce some extra notation that reveals a new form, making it possible to take into account the past behavior of the system and to analyze the structural properties. Section 3 addresses the controllability property. The specificity of linear discrete-time fractional-order systems modeled by a state-space representation leads to interesting features that are not shown by integer-order systems.

In Section 4, similar deliberations are yielded by the study of the observability property. In Section 5, we consider the case of commensurate-order systems. Finally, in Section 6, we present some numerical results corresponding to different cases of checking the controllability and observability conditions.

\section{Linear discrete-time fractional-order systems}

The discrete fractional-order difference operator $\Delta$ was defined in (Dzieliński and Sierociuk, 2005) with the zero initial time as follows:

$$
\Delta^{\alpha} x(k)=\frac{1}{h^{\alpha}} \sum_{j=0}^{k}(-1)^{j}\left(\begin{array}{l}
\alpha \\
j
\end{array}\right) x(k-j),
$$

where the fractional order $\alpha \in \mathbb{R}^{\star+}$, i.e., the set of strictly positive real numbers, $h \in \mathbb{R}^{\star+}$ is a sampling period taken equal to unity in all what follows, and $k \in \mathbb{N}$ represents the discrete time. We define

$$
\left(\begin{array}{l}
\alpha \\
j
\end{array}\right)= \begin{cases}1 & \text { for } j=0 \\
\frac{\alpha(\alpha-1) \ldots(\alpha-j+1)}{j !} & \text { for } j>0 .\end{cases}
$$

Let us consider now the traditional discrete-time statespace model of integer order, i.e., when $\alpha$ is equal to unity:

$$
\begin{aligned}
x(k+1) & =A x(k)+B u(k), \quad x(0)=x_{0}, \\
y(k) & =C x(k),
\end{aligned}
$$

where $u(k) \in \mathbb{R}^{m}$ is the input vector, $y(k) \in \mathbb{R}^{q}$ is the output vector, and $x(k) \in \mathbb{R}^{n}$ is the state vector:

$$
x(k)=\left[\begin{array}{llll}
x_{1}(k) & x_{2}(k) & \ldots & x_{n}(k)
\end{array}\right]^{T} .
$$

Its initial value is denoted by $x_{0}=x(0)$.

The first-order difference for $x(k+1)$ is defined as

$$
\Delta^{1} x(k+1)=x(k+1)-x(k) .
$$


Therefore, using Eqn. (3a) we deduce that

$$
\begin{aligned}
\Delta^{1} x(k+1) & =A x(k)+B u(k)-x(k) \\
& =A_{d} x(k)+B u(k),
\end{aligned}
$$

where $A_{d}=A-I_{n}$ and $I_{n}$ is the identity matrix.

A generalization of this integer-order difference to a non-integer-order (or fractional-order) difference was addressed in (Dzieliński and Sierociuk, 2005; Lakshmikantham, 1988). This research was conducted to construct a linear discrete-time fractional-order state-space model, using the equations

$$
\Delta^{\alpha} x(k+1)=A_{d} x(k)+B u(k), \quad x(0)=x_{0} .
$$

In this model the differentiation order $\alpha$ is taken the same for all the state variables $x_{i}(k), i=1, \ldots, n$. This is referred to as a commensurate order. Besides, from Eqn. (1) we have

$$
\Delta^{\alpha} x(k+1)=x(k+1)+\sum_{j=1}^{k+1}(-1)^{j}\left(\begin{array}{l}
\alpha \\
j
\end{array}\right) x(k-j+1) .
$$

Substituting (6) into (5) yields

$$
\begin{aligned}
& x(k+1) \\
& \quad=A_{d} x(k)-\sum_{j=1}^{k+1}(-1)^{j}\left(\begin{array}{l}
\alpha \\
j
\end{array}\right) x(k-j+1)+B u(k) .
\end{aligned}
$$

Set $c_{j}=(-1)^{j}\left(\begin{array}{l}\alpha \\ j\end{array}\right)$. Equation (7) can be rewritten as

$$
\begin{aligned}
x(k+1)= & \left(A_{d}-c_{1} I_{n}\right) x(k) \\
& -\sum_{j=2}^{k+1} c_{j} x(k-j+1)+B u(k) .
\end{aligned}
$$

Let us now write

$$
\begin{aligned}
& A_{0}=A_{d}-c_{1} I_{n}, \\
& A_{1}=-c_{2} I_{n}, \\
& A_{2}=-c_{3} I_{n},
\end{aligned}
$$

and further, for all $j>0$,

$$
A_{j}=-c_{j+1} I_{n}
$$

This leads to

$$
\begin{aligned}
x(k+1)= & A_{0} x(k)+A_{1} x(k-1)+A_{2} x(k-2) \\
& +\cdots+A_{k} x(0)+B u(k) .
\end{aligned}
$$

This description can be extended to the case of noncommensurate fractional-order systems modeled in (Dzieliński and Sierociuk, 2006):

$$
\Delta^{\Upsilon} x(k+1)=A_{d} x(k)+B u(k),
$$

$$
x(k+1)=\Delta^{\Upsilon} x(k+1)+\sum_{j=1}^{k+1} A_{j} x(k-j+1),
$$

where

$$
\Delta^{\Upsilon} x(k+1)=\left[\begin{array}{c}
\Delta^{\alpha_{1}} x_{1}(k+1) \\
\vdots \\
\Delta^{\alpha_{n}} x_{n}(k+1)
\end{array}\right],
$$

in which $\alpha_{i} \in \mathbb{R}^{\star+}, i=1,2, \ldots$ denote any fractional orders, and for each $j=1,2, \ldots$ we let

$$
A_{j}=\operatorname{diag}\left\{-(-1)^{j+1}\left(\begin{array}{c}
\alpha_{i} \\
j+1
\end{array}\right), i=1, \ldots, n\right\} .
$$

Using (10) and (11), we obtain the state equation

$$
x(k+1)=\sum_{j=0}^{k} A_{j} x(k-j)+B u(k), \quad x(0)=x_{0} .
$$

In this model, $A_{j}$ is given by (9) in the case of a commensurate fractional-order and by (11) in the case of a non-commensurate fractional-order.

Remark 1. The model described by (12) can be classified as a discrete-time system with a time-delay in the state, whereas the models addressed in (Boukas, 2006; Debeljković et al, 2002; Peng Yang et al, 2003) assume a finite constant number of steps of time-delays. Instead, the system (12) has a varying number of steps of time-delays, equal to $k$, i.e., increasing with time.

Define

$$
G_{k}= \begin{cases}I_{n} & \text { for } k=0, \\ \sum_{j=0}^{k-1} A_{j} G_{k-1-j} & \text { for } k \geq 1 .\end{cases}
$$

In an explicit way, we have

$$
\begin{aligned}
G_{0} & =I_{n}, \\
G_{1} & =\sum_{j=0}^{0} A_{j} G_{1-1-j}=A_{0} G_{0}=A_{0}, \\
G_{2} & =\sum_{j=0}^{1} A_{j} G_{1-j}=A_{0} G_{1}+A_{1} G_{0}=A_{0}^{2}+A_{1} .
\end{aligned}
$$

We thus conclude that any $G_{k}$ can be expressed equivalently either by a recurrent sum made of products $A_{j} G_{k-1-j}$ or by a recurrent sum made of products of the $A_{j}$ exclusively.

Theorem 1. The solution to (12) is given by

$$
x(k)=G_{k} x(0)+\sum_{j=0}^{k-1} G_{k-1-j} B u(j) .
$$


Proof. In Step 1, by virtue of (12) and (13), the value of the state is

$$
\begin{aligned}
x(1) & =\sum_{j=0}^{0} A_{j} x(0-j)+B u(0) \\
& =A_{0} x(0)+B u(0)=G_{1} x(0)+G_{0} B u(0) .
\end{aligned}
$$

In Step 2 we have

$$
\begin{aligned}
x(2) & =\sum_{j=0}^{1} A_{j} x(1-j)+B u(1) \\
& =\left(A_{0}^{2}+A_{1}\right) x(0)+A_{0} B u(0)+B u(1) \\
& =G_{2} x(0)+G_{1} B u(0)+G_{0} B u(1) .
\end{aligned}
$$

The property is thus satisfied in Step 2. Assume that it is true in Step $k$, i.e., that (14) is satisfied. In order to complete the demonstration of this property by induction, we have to prove that it is true in Step $k+1$.

From (12) we obtain

$$
\begin{aligned}
x(k+1) & =\sum_{j=0}^{k} A_{j} x(k-j)+B u(k) \\
& =\sum_{j=0}^{k-1} A_{j} x(k-j)+A_{k} x(0)+B u(k) .
\end{aligned}
$$

In the last equation, $x(k-j)$ can be expressed similarly to $x(k)$ since the property considered is assumed to be true up to Step $k$. Therefore, we have (introducing an extra index $l$ ):

$$
x(k-j)=G_{k-j} x(0)+\sum_{l=0}^{k-j-1} G_{k-j-1-l} B u(l) .
$$

Thereafter, coming back to the expression of $x(k+1)$ in (15) and substituting $x(k-j)$, we obtain

$$
\begin{aligned}
x(k+1) & \\
= & \sum_{j=0}^{k-1} A_{j}\left(G_{k-j} x(0)+\sum_{l=0}^{k-j-1} G_{k-j-1-l} B u(l)\right) \\
& \quad+A_{k} x(0)+B u(k) .
\end{aligned}
$$

This expression can be rewritten as follows:

$$
\begin{aligned}
x(k+1)= & \sum_{j=0}^{k-1} A_{j} G_{k-j} x(0)+A_{k} x(0) \\
& +\sum_{j=0}^{k-1} A_{j} \sum_{l=0}^{k-j-1} G_{k-j-1-l} B u(l) \\
& +B u(k) .
\end{aligned}
$$

Further, we get

$$
\begin{aligned}
x(k+1)= & \sum_{j=0}^{k} A_{j} G_{k-j} x(0) \\
& +\sum_{j=0}^{k-1} A_{j} \sum_{l=0}^{k-j-1} G_{k-j-1-l} B u(l) \\
& +B u(k) .
\end{aligned}
$$

The first sum becomes $G_{k+1} x(0)$, knowing that $G_{0}=I_{n}$. Besides, in the product of the last two sums, a permutation of indexes $j$ and $l$ yields an equivalent summation. Hence we obtain

$$
\begin{aligned}
x(k+1)= & G_{k+1} x(0)+\sum_{l=0}^{k-1} \sum_{j=0}^{k-l-1} A_{j} G_{k-j-1-l} B u(l) \\
& +G_{0} B u(k) .
\end{aligned}
$$

Next, this becomes

$$
x(k+1)=G_{k+1} x(0)+\sum_{l=0}^{k-1} G_{k-l} B u(l)+G_{0} B u(k) .
$$

Finally, we obtain that the property under study is satisfied in Step $k+1$ and we can state that it holds in any step:

$$
x(k+1)=G_{k+1} x(0)+\sum_{l=0}^{k} G_{k-l} B u(l) .
$$

This completes the proof.

The first part of the solution of (14) represents the free system response and the last part takes the role of the convolution sum corresponding to the forced response.

The corresponding transition matrix is defined as

$$
\Phi(k, 0)=G_{k}, \quad \Phi(0,0)=G_{0}=I_{n} .
$$

Remark 2. $\Phi(k, 0)$ exhibits the particularity of being time-varying in the sense that it is composed of a number of terms $A_{j}$ which grows with $k$. This is due to the fractional-order feature of the model, which takes into account all the past values of the states.

Theorem 2. The state transition matrix $\Phi(k, 0)$ has the following properties:

1. $\Phi(k, 0)$ is a solution of the homogeneous state equation

$$
\Phi(k+1,0)=\sum_{j=0}^{k} \mathrm{~A}_{j} \Phi(k-j, 0), \quad \Phi(0,0)=I_{n} .
$$


2. The semi-group property is not satisfied:

$$
\Phi\left(k_{2}, 0\right) \neq \Phi\left(k_{2}, k_{1}\right) \Phi\left(k_{1}, 0\right), \quad \forall k_{2}>k_{1}>0 .
$$

Proof. (Part 1) From (23) we deduce

$$
\Phi(k, 0)=\sum_{j=0}^{k-1} A_{j} \Phi(k-1-j, 0) .
$$

Then we directly have

$$
\Phi(k+1,0)=\sum_{j=0}^{k} A_{j} \Phi(k-j, 0) .
$$

(Part 2) Since, by definition, we have

$$
\begin{aligned}
\Phi\left(k_{1}, 0\right) & =\sum_{j=0}^{k_{1}-1} A_{j} \Phi\left(k_{1}-1-j, 0\right), \\
\Phi\left(k_{2}, 0\right) & =\sum_{j=0}^{k_{2}-1} A_{j} \Phi\left(k_{2}-1-j, 0\right), \\
\Phi\left(k_{2}, k_{1}\right) & =\sum_{j=k_{1}}^{k_{2}-1} A_{j} \Phi\left(k_{2}-1-j, 0\right),
\end{aligned}
$$

it is can be easily checked that

$$
\Phi\left(k_{2}, k_{1}\right) \Phi\left(k_{1}, 0\right) \neq \Phi\left(k_{2}, 0\right) .
$$

\section{Reachability and controllability}

In this section we discuss a fundamental question for dynamic systems modeled by (12) in the case of a noncommensurate fractional order. This question is to determine whether it is possible to transfer the state of the system from a given initial value to any other state. We attempt below to extend two concepts of state reachability (or controllability-from-the-origin) and controllability (or controllability-to-the-origin) to the present case. We are interested in completely state reachable and controllable systems.

Definition 1. The linear discrete-time fractional-order system modeled by (12) is reachable if it is possible to find a control sequence such that an arbitrary state can be reached from the origin in a finite time.

Definition 2. The linear discrete-time fractional-order system modeled by (12) is controllable if it is possible to find a control sequence such that the origin can be reached from any initial state in a finite time.

Definition 3. For the linear discrete-time fractional-order system modeled by (12) we define the following:
1. The controllability matrix:

$$
\mathcal{C}_{k}=\left[\begin{array}{lllll}
G_{0} B & G_{1} B & G_{2} B & \cdots & G_{k-1} B
\end{array}\right] .
$$

2. The reachability Gramian:

$$
W_{r}(0, k)=\sum_{j=0}^{k-1} G_{j} B B^{T} G_{j}^{T}, \quad k \geq 1 .
$$

It is easy to show that $W_{r}(0, k)=\mathcal{C}_{k} \mathcal{C}_{k}^{T}$.

3. The controllability Gramian, provided that $A_{0}$ is non-singular:

$$
W_{c}(0, k)=G_{k}^{-1} W_{r}(0, k) G_{k}^{-T}, \quad k \geq 1 .
$$

Note that $G_{1}=A_{0}$, and the existence of $W_{r}(0,1)$ imposes $A_{0}$ to be nonsingular. However, this is not that restrictive a condition because a discrete model is often obtained by sampling a continuous one. Thus, in the remainder of this paper we assume that $A_{0}$ is non-singular.

Theorem 3. The linear discrete-time fractional-order system modeled by (12) is reachable if and only if there exists a finite time $K$ such that $\operatorname{rank}\left(\mathcal{C}_{K}\right)=n$ or, equivalently, $\operatorname{rank}\left(W_{r}(0, K)\right)=n$. Furthermore, the input sequence

$$
\mathcal{U}_{K}=\left[\begin{array}{llll}
u^{T}(K-1) & u^{T}(K-2) & \ldots & u^{T}(0)
\end{array}\right]^{T}
$$

that transfers $x_{0}=0$ at $k=0$ to $x_{f} \neq 0$ at $k=K$ is given by

$$
\mathcal{U}_{K}=\mathcal{C}_{K}^{T} W_{r}^{-1}(0, K) x_{f}
$$

Proof. (Sufficiency) Let $x_{f}$ be the final state to be reached. From (14) we have

$$
x_{f}(k)=G_{k} x_{0}+\sum_{j=0}^{k-1} G_{k-1-j} B u(j) .
$$

With $x_{0}=0$, this gives

$$
x_{f}(k)=\mathcal{C}_{k} \mathcal{U}_{k},
$$

where $\mathcal{U}_{k}=\left[\begin{array}{llll}u^{T}(k-1) & u^{T}(k-2) & \ldots & u^{T}(0)\end{array}\right]^{T}$. Equation (28) has a unique solution $\mathcal{U}_{k}$ at Step $k=K$ if $\operatorname{rank}\left(\mathcal{C}_{K}\right)=n$. Besides, we have $W_{r}(0, K)=\mathcal{C}_{K} \mathcal{C}_{K}^{T}$. Hence, if $\operatorname{rank}\left(\mathcal{C}_{K}\right)=n$, then $\operatorname{rank}\left(W_{r}(0, K)\right)=n$. It follows that $W_{r}(0, K)$ is a positive definite non-singular matrix. At Step $k=K$ we have

$$
x_{f}(K)=\mathcal{C}_{K} \mathcal{U}_{K}
$$

Substituting (27) into (29), we get

$$
x_{f}(K)=\mathcal{C}_{K} \mathcal{C}_{K}^{T} W_{r}^{-1}(0, K) x_{f}=x_{f} .
$$


We conclude that the system (12) is reachable.

(Necessity) This part is by contradiction. Assume that the system (19) is reachable but $\operatorname{rank}\left(\mathcal{C}_{k}\right)=n$ for any $k>0$, which implies that the rows of $\mathcal{C}_{k}$ are linearly dependent for any $k>0$. It results that there exists a non-zero constant $1 \times n$ row vector $v$ such that

$$
v \mathcal{C}_{k}=0
$$

From (28) we have

$$
v x_{f}(k)=v \mathcal{C}_{k} \mathcal{U}_{k}=0,
$$

which implies that $x_{f}(k)=0$ for any $k>0$, i.e., the system is not reachable. This is a contradiction, which completes the proof.

Remark 3. In the case of an integer order, it is well known that the rank of $\mathcal{C}_{k}$ cannot increase for any $k \geq$ $n$. This results from the Cayley-Hamilton theorem. On the contrary, in the case of the linear discrete-time noncommensurate fractional-order system (12), the rank of $\mathcal{C}_{k}$ can increase for values of $k \geq n$. In other words, it is possible to reach the final state $x_{f}$ in a number of steps greater than $n$. This is due to the nature of the elements $G_{k}$ which build up the controllability matrix $\mathcal{C}_{k}$ and which exhibit the particularity of being time-varying, in the sense that they are composed of a number of terms $A_{j}$ that grows with $k$, as has already mentioned in Remark 2 . The full rank of $\left(\mathcal{C}_{k}\right)$ can be reached at some Step $k=K$ equal to or greater than $n$.

Theorem 4. The linear discrete-time fractional-order system modeled by (12) is controllable if and only if there exists a finite time $K$ such that $\operatorname{rank}\left(W_{c}(0, K)\right)=$ $n$. Furthermore, an input sequence $\mathcal{U}_{K}=\left[u^{T}(K-\right.$ 1) $\left.u^{T}(K-2) \quad \ldots \quad u^{T}(0)\right]^{T}$ that transfers $x_{0} \neq 0$ at $k=0$ to $x_{f}=0$ at $k=K$ is given by

$$
\mathcal{U}_{K}=-\mathcal{C}_{K}^{T} G_{K}^{-T} W_{c}^{-1}(0, K) x_{0} .
$$

Proof. (Sufficiency) Let $x_{f}=0$ be the final state to be reached at some finite time $K$ from an initial state $x_{0} \neq 0$. From (14) we have

$$
x_{f}=G_{K} x_{0}+\mathcal{C}_{K} \mathcal{U}_{K}=0,
$$

which gives

$$
x_{0}=-G_{K}^{-1} \mathcal{C}_{K} \mathcal{U}_{K} .
$$

If we get $\operatorname{rank}\left(W_{c}(0, K)\right)=n$ for some $K$, then $W_{c}^{-1}(0, K)$ exists. Substituting (30) into (31) yields

$$
\begin{aligned}
x_{0} & =G_{K}^{-1} \mathcal{C}_{K} \mathcal{C}_{K}^{T} G_{K}^{-T} W_{c}^{-1}(0, K) x_{0} \\
& =W_{c}(0, K) W_{c}^{-1}(0, K) x_{0}=x_{0} .
\end{aligned}
$$

(Necessity) The proof is by contradiction. Assume that (12) is controllable but $\operatorname{rank}\left(W_{c}(0, k)\right)<n$ for any $k>0$. Since $G_{k}$ is full rank for $k \geq 0$, we have $\operatorname{rank}\left(W_{c}(0, k)\right)=\operatorname{rank}\left(W_{r}(0, k)\right)=\operatorname{rank}\left(\mathcal{C}_{k}\right)$. It follows that there exists a non-zero constant $1 \times n$ row vector $w$ such that

$$
w \mathcal{C}_{k}=0
$$

Since $x_{f}=0$, from (14) we have

$$
w x_{f}=w G_{k} x_{0}+w \mathcal{C}_{k} \mathcal{U}_{k}=0 .
$$

This implies that $w G_{k} x_{0}=0$, i.e., $x_{0}=0$. This is a contradiction, which completes the proof.

\section{Observability}

In this section we aim at extending the concept of observability to the system of equations (12) and (3b), in the case of a non-commensurate fractional order. We are interested in completely state observable systems.

Definition 4. The linear discrete-time fractional-order system modeled by (12) and ( $3 b)$ is observable at time $k=0$ if and only if there exits some $K>0$ such that the state $x_{0}$ at time $k=0$ can be uniquely determined from the knowledge of $u_{k}, y_{k}, k \in[0, K]$.

Definition 5. For the linear discrete-time fractional-order system modeled by (12) and (3b) we define the following:

1. The observability matrix:

$$
\mathcal{O}_{k}=\left[\begin{array}{c}
C G_{0} \\
C G_{1} \\
C G_{2} \\
\vdots \\
C G_{k-1}
\end{array}\right]
$$

2. The observability Gramian:

$$
W_{o}(0, k)=\sum_{j=0}^{k-1} G_{j}^{T} C^{T} C G_{j} .
$$

It is easy to show that $W_{o}(0, k)=\mathcal{O}_{k}^{T} \mathcal{O}_{k}$.

Theorem 5. The linear discrete-time fractional-order system modeled by (12) and (3b) is observable if and only if there exists a finite time $K$ such that $\operatorname{rank}\left(\mathcal{O}_{K}\right)=n$ or, equivalently, $\operatorname{rank}\left(W_{o}(0, K)\right)=n$. Furthermore, the initial state $x_{0}$ at $k=0$ is given by

$$
x_{0}=W_{o}^{-1}(0, K) \mathcal{O}_{K}^{T}\left[\tilde{\mathcal{Y}}_{K}-\mathcal{M}_{K} \tilde{\mathcal{U}}_{K}\right]
$$


with

$$
\begin{aligned}
& \tilde{\mathcal{U}}_{K}=\left[\begin{array}{llll}
u^{T}(0) & u^{T}(1) & \ldots & u^{T}(K-1)
\end{array}\right]^{T}, \\
& \tilde{\mathcal{Y}}_{K}=\left[\begin{array}{llll}
y^{T}(0) & y^{T}(1) & \ldots & y^{T}(K-1)
\end{array}\right]^{T}, \\
& \mathcal{M}_{K} \\
& =\left[\begin{array}{ccccc}
0 & 0 & \ldots & 0 & 0 \\
C G_{0} B & 0 & \ldots & 0 & 0 \\
C G_{1} B & C G_{0} B & \ldots & 0 & 0 \\
C G_{2} B & C G_{1} B & \ldots & 0 & 0 \\
\vdots & \vdots & \vdots & \vdots & \vdots \\
C G_{K-2} B & C G_{K-3} B & \ldots & C G_{0} B & 0
\end{array}\right]
\end{aligned}
$$

Proof. (Sufficiency) From the output equation (3b) and Eqn. (14) we have

$$
\begin{aligned}
& y(0)=C x(0)=C G_{0} x(0), \\
& y(1)=C x(1)=C G_{1} x(0)+C G_{0} B u(0), \\
& y(2)=C x(2)=C G_{2} x(0)+C G_{1} B u(0)+C G_{0} B u(1),
\end{aligned}
$$

and, at last,

$$
\begin{aligned}
y(k-1)= & C x(k-1) \\
= & C G_{k-1} x(0)+C G_{k-2} B u(0) \\
& +C G_{k-3} B u(1)+\ldots \\
& +C G_{0} B u(k-2) .
\end{aligned}
$$

The above relations can be written in the following condensed form:

$$
\tilde{\mathcal{Y}}_{k}=\mathcal{O}_{k} x(0)+\mathcal{M}_{k} \tilde{\mathcal{U}}_{k},
$$

where

$$
\begin{aligned}
\tilde{\mathcal{U}}_{k} & =\left[\begin{array}{llll}
u^{T}(0) & u^{T}(1) & \ldots & u^{T}(k-1)
\end{array}\right]^{T}, \\
\tilde{\mathcal{Y}}_{k} & =\left[\begin{array}{llll}
y^{T}(0) & y^{T}(1) & \ldots & y^{T}(k-1)
\end{array}\right]^{T} .
\end{aligned}
$$

At time $k=K$, we can write

$$
\tilde{\mathcal{Y}}_{K}=\mathcal{O}_{K} x(0)+\mathcal{M}_{K} \tilde{\mathcal{U}}_{K} .
$$

It follows that

$$
\mathcal{O}_{K} x(0)=\tilde{\mathcal{Y}}_{K}-\mathcal{M}_{K} \tilde{\mathcal{U}}_{K}
$$

Then

$$
\mathcal{O}_{K}^{T} \mathcal{O}_{K} x(0)=\mathcal{O}_{K}^{T}\left(\tilde{\mathcal{Y}}_{K}-\mathcal{M}_{K} \tilde{\mathcal{U}}_{K}\right)
$$

which becomes

$$
W_{o}(0, K) x(0)=\mathcal{O}_{K}^{T}\left(\tilde{\mathcal{Y}}_{K}-\mathcal{M}_{K} \tilde{\mathcal{U}}_{K}\right) .
$$

If $\operatorname{rank}\left(\mathcal{O}_{K}\right)=n$ or, equivalently, if $\operatorname{rank}\left(W_{o}(0, K)\right)=$ $n$, then $W_{o}(0, K)$ is positive definite. Consequently, we obtain

$$
x(0)=W_{o}^{-1}(0, K) \mathcal{O}_{K}^{T}\left(\tilde{\mathcal{Y}}_{K}-\mathcal{M}_{K} \tilde{\mathcal{U}}_{K}\right) .
$$

(Necessity) The proof is by contradiction. Assume that the system of equations (12) and (3b) is observable but $\operatorname{rank}\left(\mathcal{O}_{k}\right)<n$ for any $k>0$. Then the columns of $\mathcal{O}_{k}$ are linearly dependent for any $k>0$, i.e., there exists a non-zero constant column $n \times 1$ vector $z$ such that

$$
\mathcal{O}_{k} z=0
$$

Let us choose $x(0)=z$. From the relation

$$
\tilde{\mathcal{Y}}_{k}=\mathcal{O}_{k} x(0)+\mathcal{M}_{k} \tilde{\mathcal{U}}_{k},
$$

we deduce

$$
\mathcal{O}_{k} z=\tilde{\mathcal{Y}}_{k}-\mathcal{M}_{k} \tilde{\mathcal{U}}_{k}=0 \text {. }
$$

Hence the initial state $x(0)=z$ is not detected. This is in contradiction with the assumption that the system of equations (12) and (3b) is observable. This completes the proof.

Remark 4. From the Cayley-Hamilton theorem, it is well known that for integer-order systems the rank of the observability matrix $\mathcal{O}_{k}$ cannot increase at Step $k \geq n$. Here, too, it is remarkable that this is not true in the case of the discrete-time non-commensurate fractional-order system of (12) and (3b). Indeed, $\operatorname{rank}\left(\mathcal{O}_{k}\right)$ can increase for values $k \geq n$. We can state that the observability of this type of systems can possibly be obtained in a number of steps greater than $n$. This is due to the same reasons as those exposed above in Remark 3 for controllability. In (Dzieliński and Sierociuk, 2006), the observability condition for the discrete-time fractional-order system as modeled in (12), with a non-commensurate order, is that the rank of $\mathcal{O}_{k}$ should be equal to $n$ at most at Step $k=n$. Our result shows that the full rank of $\left(\mathcal{O}_{k}\right)$ can be reached at some step $k=K$ greater than $n$. This can be considered as an extension of the previous result in (Dzieliński and Sierociuk, 2006).

Remark 5. The property of reconstructibility (Antsaklis and Michel, 1997) can also be studied in this case. Note that if $A_{0}$ is non-singular, then observability and reconstructibility are equivalent.

\section{Commensurate fractional-order case}

In this section we address the particular case of commensurate fractional-order systems. The terms $A_{j}$ are expressed by (9). It is clear then that the matrices $G_{k}$ defined by (13) are polynomials in $A_{0}$, i.e.,

$$
G_{k}=A_{0}^{k}+\beta_{1_{k}} A_{0}^{k-1}+\beta_{2_{k}} A_{0}^{k-2}+\cdots+\beta_{k_{k}} I_{n},
$$


where the real coefficients $\beta_{j_{k}}$ are calculated from the coefficients $c_{j}$. In particular, we have

$$
G_{n}=A_{0}^{n}+\beta_{1_{n}} A_{0}^{n-1}+\beta_{2_{n}} A_{0}^{n-2}+\cdots+\beta_{n_{n}} I_{n} .
$$

From the Cayley-Hamilton theorem, $A_{0}^{n}$ is a linear combination of $A_{0}^{n-1}, A_{0}^{n-2}, \ldots, I_{n}$. We deduce that $G_{k+n}$, for all $k \geq 0$ are linearly dependent on $G_{n-1}, G_{n-2}, \ldots, I_{n}$. This implies the following results:

Corollary 1. The linear discrete-time fractional-order system modeled by (12) and ( $3 b)$ in the commensurate case is reachable if and only if $\operatorname{rank}\left(\mathcal{C}_{n}\right)=n$ or, equivalently, $\operatorname{rank}\left(W_{r}(0, n)\right)=n$. On the other hand, this system is controllable if and only if $\operatorname{rank}\left(W_{c}(0, n)\right)=n$.

Corollary 2. The linear discrete-time fractional-order system modeled by (12) and (3b) in the commensurate case is observable if and only if $\operatorname{rank}\left(\mathcal{O}_{n}\right)=n$ or, equivalently, $\operatorname{rank}\left(W_{o}(0, n)\right)=n$.

Remark 6. We therefore observe that the contollability and observability criteria for the commensurate fractionalorder case are similar to those of the integer-order case, in the sense that if a state cannot be reached in $n$ steps, then it is not reachable at all, and that if an initial state cannot be deduced from $n$ steps of input-output data, then it is not observable at all. The result given in (Dzieliński and Sierociuk, 2006) which states that a necessary and sufficient condition for the discrete-time fractional-order system as modeled in (12) and (3b) to be observable is that the rank of $\mathcal{O}_{k}$ should be equal to $n$ at most at Step $k=n$ is true only in the case of commensurate fractional-order systems.

\section{Numerical examples}

6.1. Reachability. Consider the following discretetime non-commensurate fractional-order of dimension $n=4$, with

$$
\begin{gathered}
\alpha_{1}=0.2, \quad \alpha_{2}=0.3, \quad \alpha_{3}=0.6, \quad \alpha_{4}=0.7, \\
A_{d}=\left[\begin{array}{cccc}
-0.7 & -1 & 4 & -0.5 \\
1 & -1.6 & 1.5 & 0.8 \\
2 & -3 & -0.1 & 2.5 \\
-0.8 & 0.7 & 1.8 & -0.4
\end{array}\right], \\
B=\left[\begin{array}{llll}
10 & 10 & 10 & 10
\end{array}\right]^{T} .
\end{gathered}
$$

We determined $\operatorname{rank}\left(\mathcal{C}_{k}\right)$ over a set of $N=20$ samples. We found $\operatorname{rank}\left(\mathcal{C}_{k}\right)=4$ at $K=5$ and

$$
\mathcal{C}_{K}=\left[\begin{array}{lllll}
10.00 & 20.00 & 40.80 & 84.90 & 173.31 \\
10.00 & 20.00 & 41.05 & 84.77 & 175.66 \\
10.00 & 20.00 & 41.20 & 84.63 & 177.03 \\
10.00 & 20.00 & 41.05 & 85.12 & 174.78
\end{array}\right]
$$

We chose the final state

$$
x_{f}=\left[\begin{array}{llll}
1 & -0.5 & 3 & 0.3
\end{array}\right]^{T} .
$$

The input sequence that permitted to transfer the state from the origin to $x_{f}$ according to (27) is

$$
\mathcal{U}_{K}=\left[\begin{array}{lllll}
30.31 & 60.61 & 210.91 & -64.38 & -26.85
\end{array}\right]^{T} .
$$

Table 1 gives the values of the state variables at each step. We see that the final state has been reached within a number of steps of the input data sequence greater than the system dimension. This comes up to be a particu-

Table 1. Values of the state variables in the transfer steps.

\begin{tabular}{|c|c|c|c|c|}
\hline$k$ & $x_{1}(k)$ & $x_{2}(k)$ & $x_{3}(k)$ & $x_{4}(k)$ \\
\hline \hline 0 & 0 & 0 & 0 & 0 \\
1 & -268.49 & -268.49 & -268.49 & -268.49 \\
2 & -1180.76 & -1180.76 & -1180.76 & -1180.76 \\
3 & -273.93 & -280.65 & -284.67 & -280.65 \\
4 & -81.96 & -94.43 & -100.46 & -103.96 \\
5 & 1.00 & -0.50 & 3.00 & 0.30 \\
\hline
\end{tabular}

larity of discrete non-commensurate fractional-order systems. This is not satisfied in the case of discrete commensurate fractional-order systems for which the full rank, $n$, if it can be reached, cannot be reached beyond the number of steps $K=n$. The states show some values of large magnitude. Nevertheless, the objective is reached.

6.2. Observability. We considered the system with $\alpha_{1}=0.2, \alpha_{2}=0.3, \alpha_{3}=0.6, \alpha_{4}=0.7$,

$$
\begin{gathered}
A_{d}=\left[\begin{array}{cccc}
-0.4 & -1 & 4 & -0.5 \\
1 & 5 & 1.5 & 0.8 \\
2 & -3 & -5.9 & 2.5 \\
-0.8 & 0.7 & 1.8 & -1.5
\end{array}\right] \\
B=\left[\begin{array}{l}
1 \\
1 \\
1 \\
1
\end{array}\right] \\
C=\left[\begin{array}{lll}
1 & 1 & 1
\end{array}\right]
\end{gathered}
$$

We determined $\operatorname{rank}\left(\mathcal{O}_{k}\right)$ over a set of $N=20$ samples. We found $\operatorname{rank}\left(\mathcal{O}_{k}\right)=4$ at $K=5$ and

$$
\mathcal{O}_{K}=\left[\begin{array}{cccc}
1.00 & 1.00 & 1.00 & 1.00 \\
2.00 & 2.00 & 2.00 & 2.00 \\
4.08 & 4.10 & 4.12 & 4.10 \\
8.45 & 8.459 & 8.33 & 8.51 \\
17.06 & 17.95 & 18.34 & 17.09
\end{array}\right] \text {. }
$$


We chose the following input sequence over 5 steps:

$$
\tilde{\mathcal{U}}_{K}=\left[\begin{array}{lllll}
1 & -0.2 & 5 & 10 & -0.6
\end{array}\right]^{T} \text {. }
$$

In this example, we see that the second row of $\mathcal{O}_{K}$ is the doubled first row. The output sequence must be chosen so as to take into account this dependence. Let us denote by $\tilde{\mathcal{Y}}_{K}^{*}$ the zero-input response of the system

$$
\begin{aligned}
\tilde{\mathcal{Y}}_{K}^{*} & =\tilde{\mathcal{Y}}_{K}-\mathcal{M}_{k} * \tilde{\mathcal{U}}_{K} \\
& =\left[\begin{array}{lllll}
y^{*}(0) & y^{*}(1) & y^{*}(2) & y^{*}(3) & y^{*}(4)
\end{array}\right]^{T} .
\end{aligned}
$$

The output sequence $\tilde{\mathcal{Y}}_{K}$ must be then chosen so as to get $y^{*}(1)=2 y^{*}(0)$.

A candidate output sequence is, e.g.,

$$
\tilde{\mathcal{Y}}_{K}=\left[\begin{array}{lllll}
1 & 6 & -2 & 7 & 3
\end{array}\right]^{T} \text {. }
$$

According to (34), the initial state

$$
x_{0}=\left[\begin{array}{llll}
1.22 & -3.27 & 1.63 & 0.41
\end{array}\right]^{T}
$$

is detected. The corresponding determinant of the observability gramian is

$$
\operatorname{det}\left[W_{o}(0, K)\right]=4.97 \times 10^{-5} .
$$

The singular value decomposition of $W_{o}(0, K)$ gives

$$
\Sigma=\operatorname{diag}\left(1613.86,0.38,9.80 \times 10^{-4}, 8.34 \times 10^{-5}\right) .
$$

We observe that, except the first one, the singular values are quite small: the corresponding states are weakly observable. The plot of the simulated output, starting from the detected initial state $x_{0}$, is illustrated in Fig. 1. The simulated output sequence is identical to the chosen initial output sequence. It is possible to consider other examples with stronger observability. For this purpose, let us consider the same example in which the output matrix $C$ is successively changed into

$$
C=\left[\begin{array}{llll}
5 & 5 & 5 & 5
\end{array}\right]
$$

and

$$
C=\left[\begin{array}{llll}
10 & 10 & 10 & 10
\end{array}\right]
$$

The determinant of the observability Gramian takes the values $\operatorname{det}\left[W_{o}(0, K)\right]=19.422$ and $\operatorname{det}\left[W_{o}(0, K)\right]=$ 4972 , respectively. This shows that the state variables may become strongly observable.

\section{Conclusion}

In this paper we investigated the structural properties of the controllability and observability of linear discrete-time

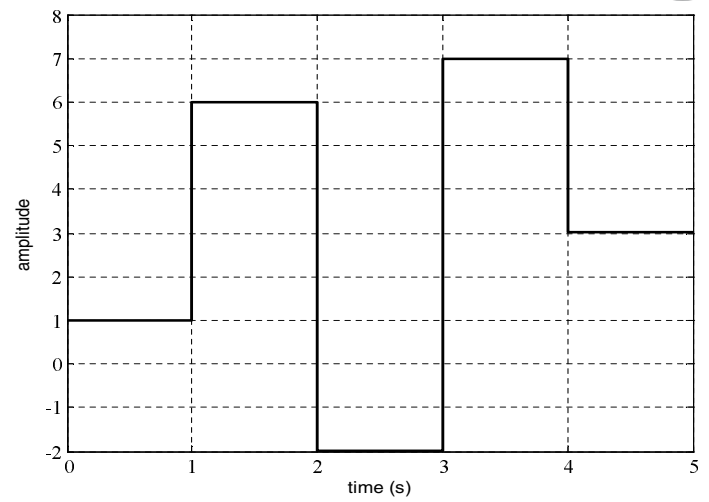

Fig. 1. Computed values of the output sequence.

fractional-order systems. We established a new formulation of the state-space equation and showed that this new formulation makes it possible to analyze effectively these properties. In addition, it reveals new controllability and observability conditions in both cases of noncommensurate and commensurate fractional-orders. We verified the theoretical results stated in this paper with suitable numerical examples.

\section{Acknowledgments}

The authors would like to thank the anonymous reviewers for their numerous constructive remarks that have improved this paper.

\section{References}

Antsaklis P.J. and Michel A.N. (1997). Linear Systems, McGraw-Hill, New York.

Åström K. J. and Wittenmark B. (1996). Computer-Controlled Systems, Theory and Design, 3rd Ed., Prentice Hall Inc., New Jersey.

Axtell M. and Bise E. M. (1990). Fractional calculus applications in control systems, Proceedings of the IEEE $1990 \mathrm{Na}$ tional Aerospace and Electronics Conference, New York, USA, pp. 536-566.

Battaglia J. L., Cois O., Puigsegur L. and Oustaloup A. (2001). Solving an inverse heat conduction problem using a noninteger identified model, International Journal of Heat and Mass Transfer, 44(14): 2671-2680.

Bettayeb M. and Djennoune S. (2006). A note on the controllability and the observability of fractional dynamical systems, Proceedings of the 2nd IFAC Workshop on Fractional Differentiation and its Workshop Applications, Porto, Portugal, pp. 506-511.

Boukas E.K. (2006). Discrete-time systems with time-varying time delay: Stability and stabilizability, Mathematical Problems in Engineering, bf 2006 (ID42489): 1-10.

Cois O., Oustaloup A., Battaglia E. and Battaglia J.L. (2002). Non integer model from modal decomposition for time domain identification, 41st IEEE CDC'2002 Tutorial Workshop 2, Las Vegas, USA. 
Debeljković D. Lj., Aleksendrić M., Yi-Yong N. and Zhang Q. L. (2002). Lyapunov and non-Lyapunov stability of linear discrete time delay systems, Facta Universitatis, Series: Mechanical Engineering 1(9): 1147-1160.

Dorčák L., Petras I. and Kostial I. (2000). Modeling and analysis of fractional-order regulated systems in the state-space, Procedings of International Carpathian Control Conference, High Tatras, Slovak Republic, pp. 185-188.

Dzieliński A. and Sierociuk D. (2005). Adaptive feedback control of fractional order discrete state-space systems, Proceedings of the 2005 International Conference on Computational Intelligence for Modelling, Control and Automation, and International Conference on Intelligent Agents, Web Technologies and Internet Commerce (CIMCA-IAWTIC'05), Vienna Austria, pp. 804-809.

Dzieliński A. and Sierociuk D. (2006). Observer for discrete fractional order systems, Proceedings of the 2nd IFAC Workshop on Fractional Differentiation Applications, Porto, Portugal, pp. 524-529.

Dzieliński A. and Sierociuk D. (2007). Reachability, controllability and observability of the fractional order discrete statespace system, Proceedings of the IEEE/IFAC International Conference on Methods and Models in Automation and Robotics, MMAR'2007, Szczecin, Poland, pp. 129-134.

Gorenflo R. and Mainardi F. (1997). Fractional calculus: Integral and differential equations of fractional order, in (A. Carpintieri and F. Mainardi, Eds.) Fractals and Fractional Calculus in Continuum Mechanics, Vienna, New York, Springer Verlag.

Hanyga A. (2003). Internal variable models of viscoelasticity with fractional relaxation laws, Proceddings of Design Engineering Technical Conference, Mechanical Vibration and Noise, 48395, American Society of Mechanical Engineers, Chicago, USA.

Hotzel R. and Fliess M.(1998). On linear system with a fractional derivation: Introductory theory and examples, Mathematics and Computers in Simulation 45 (3): 385-395.

Ichise M., Nagayanagi Y. and Kojima T. (1971). An analog simulation of non integer order transfer functions for analysis of electrode processes, Journal of Electroanalytical Chemistry 33(2): 253-265.

Kilbas A. A., Srivasta H. M. and Trujillo J. J. (2006). Theory and Applications of Fractional Differential Equations, Elsevier, Amsterdam.

Lakshmikantham D. T. V. (1998). Theory of Difference Equations: Numerical Methods and Applications, Academic Press, New York.

Manabe S. (1960). The non-integer integral and its application to control systems, Japanese Institute of Electrical Engineers Journal 80(860): 589-597.

Matignon D. (1994). Reprèsentation en variables d'ètat de modèles de guides d'ondes avec dèrivation fractionnaire, $\mathrm{Ph}$.D. thesis, Universitè Paris XI, France.

Matignon D., d'Andrèa Novel B., Depalle P. and Oustaloup A. (1994). Viscothermal Losses in Wind Instruments: A NonInteger Model, Academic Verlag, Berlin.
Matignon D. and d'Andrèa-Novel B. (1996). Some results on controllability and observability of finite-dimensional fractional differential systems, Proceedings of the IMACS, IEEE SMC Conference, Lille, France, pp. 952-956.

Matignon D. (1996). Stability results on fractional differential with application to control processing, Proceedings of the IAMCS, IEEE SMC Conference, Lille, France, pp. 963968.

Miller K. S. and Ross B. (1993). An Introduction to the Fractional Calculus and Fractional Differential Equations, Wiley, New York.

Mittag-Leffler G. (1904). Sur la reprèsentation analytique d'une branche uniforme d'une fonction monogène, Acta Mathematica 29: 10-181.

Oldham K. B. and Spanier J. (1974). The Fractional Calculus, Academic Press, New York.

Oustaloup A. (1983). Systèmes asservis linèaires d'ordre fractionnaire, Masson, Paris.

Oustaloup A. (1995). La Dèrivation non entière: Thèorie, synthèse et applications, Hermès, Paris.

Peng Y., Guangming X. and Long W. (2003). Controllability of linear discrete-time systems with time-delay in state, available at dean.pku.edu.cn/bksky/1999tzlwj/4.pdf.

Podlubny I. (1999). Fractional Differential Equations, Academic Press, San Diego.

Raynaud H. F., Zergainoh, A. (2000). State-space representation for fractional-order controllers, Automatica 36(7): 1017 1021.

Sabatier J., Cois O. and Oustaloup A. (2002). Commande de systèmes non entiers par placement de pôles, Deuxième Confèrence Internationale Francophone d'Automatique, CIFA, Nantes, France.

Samko S. G., Kilbas A. A. and Marichev O. I. (1993). Fractional Integrals and Derivatives: Theory and Applications, Gordon and Breach, Amsterdam.

Sierociuk D. and Dzieliński A. (2006). Fractional Kalman filter algorithm for the states, parameters and order of fractional system estimation, International Journal of Applied Mathematics and Computer Science 16(1): 129-140.

Valerio D. and Sa da Costa J. (2004). Non-integer order control of a flexible robot, Proceedings of the IFAC Workshop on Fractional Differentiation and its Applications, FDA'04, Bordeaux, France.

Vinagre B. M., Monje C. A. and Caldero A. J. (2002). Fractional order systems and fractional order actions, Tutorial Workshop 2: Fractional Calculus Applications in Automatic Control and Robotics, 41st IEEE CDC, Las Vegas, USA.

Received: 17 June 2007 Revised: 21 October 2007 